\title{
A CLASS OF SHANNON-MCMILLAN THEOREMS FOR MTH-ORDER MARKOV INFORMATION SOURCE ON GENERALIZED RANDOM SELECTION SYSTEM
}

\author{
KANGKANG WANG AND DECAI ZONG
}

\begin{abstract}
In this paper, our aim is to establish a class of Shannon-McMillan theorems for $m$ th-order nonhomogeneous Markov information source on the generalized random selection system by constructing the consistent distribution functions. As corollaries, we obtain some Shannon-McMillan theorems for $m$ th-order nonhomogeneous Markov information source and the general nonhomogeneous Markov information source. Some results which have been obtained are extended. In the proof, a new technique for studying Shannon-McMillan theorems in information theory is applied.
\end{abstract}

\section{Introduction}

Let $(\Omega, \mathscr{F}, P)$ be a probability space, $\left\{X_{n}, n \geq 0\right\}$ be an arbitrary information source defined on $(\Omega, \mathscr{F}, P)$ which takes values on the alphabet set $S=\left\{s_{1}, s_{2}, \ldots, s_{M}\right\}$ with joint distribution:

$$
P\left(X_{0}=x_{0}, \ldots, X_{n}=x_{n}\right)=p\left(x_{0}, \ldots, x_{n}\right)>0, x_{i} \in S, 0 \leq i \leq n .
$$

Let

$$
f_{n}(\omega)=-\frac{1}{n+1} \log p\left(X_{0}, \ldots, X_{n}\right),
$$

where $\log$ is natural logarithmic, $f_{n}(\omega)$ is called the relative entropy density of $\left\{X_{i}, 0 \leq i \leq n\right\}$.

If $\left\{X_{n}, n \geq 0\right\}$ be an $m$ th-order nonhomogeneous Markov information source, then as $n \geq m$,

$$
P\left(X_{n}=x_{n} \mid X_{0}=x_{0}, \ldots, X_{n-1}=x_{n-1}\right)=P\left(X_{n}=x_{n} \mid X_{n-m}=x_{n-m}, \ldots, X_{n-1}=x_{n-1}\right) .
$$

Denote

$$
q\left(i_{0}, \ldots, i_{m-1}\right)=P\left(X_{0}=i_{0}, \ldots, X_{m-1}=i_{m-1}\right),
$$

Corresponding author: Kangkang Wang.

2010 Mathematics Subject Classification. 60F15.

Key words and phrases. Generalized Shannon-Mcmillan theorem, the consistent distribution, $m$ thorder Markov information source, relative entropy density.

The work is supported by the Project of Higher Schools' Natural Science Basic Research of Jiangsu Province of China (09KJD110002). 


$$
p_{n}\left(j \mid i_{1}, \ldots, i_{m}\right)=P\left(X_{n}=j \mid X_{n-m}=i_{1}, \ldots, X_{n-1}=i_{m}\right) .
$$

$q\left(i_{0}, \ldots, i_{m-1}\right)$ is called the $m$ dimensional initial distribution. $p_{n}\left(j \mid i_{1}, \ldots, i_{m}\right), n \geq m$ are called the $m$ th-order transition probabilities, and

$$
P_{n}=\left(p_{n}\left(j \mid i_{1}, \ldots, i_{m}\right)\right)
$$

are called the $m$ th-order transition matrices. In the case,

$$
\begin{gathered}
p\left(x_{0}, \ldots, x_{n}\right)=q\left(x_{0}, \ldots, x_{m-1}\right) \prod_{k=m}^{n} p_{k}\left(x_{k} \mid x_{k-m}, \ldots, x_{k-1}\right), \\
f_{n}(\omega)=-\frac{1}{n+1}\left[\log q\left(X_{0}, \ldots, X_{m-1}\right)+\sum_{k=m}^{n} \log p_{k}\left(X_{k} \mid X_{k-m}, \ldots, X_{k-1}\right)\right] .
\end{gathered}
$$

The convergence of $f_{n}(\omega)$ in a sense ( $L_{1}$ convergence, convergence in probability, a.s. convergence) is called Shannon-McMillan theorem or entropy theorem or asymptotic equipartition property (AEP) in information. Shannon [1] first proved the AEP for convergence in probability for stationary ergodic information source with a finite alphabet set. McMillan [2] and Breiman [3] proved the AEP in $L_{1}$ and a.s. convergence, respectively, for stationary ergodic information source. Chung [4] considered the case of the countable alphabet. The AEP for general stochastic processes can be found, for example, in Barron [5] and Algoet and Cover [6]. Liu and Yang [7] have proved AEP for a class of nonhomogeneous Markov information sources.

The conception of random selection derives from gambling. We consider a sequence of Bernoulli trial, and suppose that at each trial the bettor has the free choice of whether or not to bet. A theorem on gambling system asserts that under any non-anticipative system the successive bets form a sequence of Bernoulli trial with unchanged probability for success. The importance of this statement was recognized by von Mises, who introduced the impossibility of a successful gambling system as a fundamental axiom (see [8], [9]). This topic was discussed still further by Kolmogrov (see [10]) and Liu and Wang (see [11] and [12]).

Many of practical information source, such as language and image information, are often $m$ th-order Markov information source, and always nonhomogeneous. Hence it is of importance to study the AEP for the $m$ th-order nonhomogeneous Markov information source in the information theory. The purpose of this paper is to generalize Shannon-McMillan theorems for $m$ th-order nonhomogeneous Markov information source to the case of the generalized random selection system by constructing the consistent distribution functions and nonnegative sup-martingale. As corollaries, we obtain some Shannon-McMillan theorems for $m$ thorder Markov chain and the general Markov chain. Some results of Liu and Yang (see [13] and 
[7]) are extended. In the proof, we apply a new technique to studying the strong limit theorems for entropy density in information theory. Afterward, many scholars (see [15]-[33]) have studied all kinds of stochastic processes and some limit properties with their applications for $m$ th-order nonhomogeneous Markov chains on the generalized gambling system.

In order to extend the conception of random selection, which is the crucial part of the gambling system, we first give a set of real-valued functions $f_{n}\left(x_{0}, \ldots, x_{n}\right)$ defined on $S^{n+1}(n=$ $1,2, \ldots)$, which will be called the generalized selection functions if they take values in an arbitrary real interval of $[a, b],(a, b \in R)$ (The traditional random selection system [12] takes values in the set of $\{0,1\})$. We let

$$
\begin{aligned}
Y_{0} & =y(y \text { is an arbitrary real number }) \\
Y_{n+1} & =f_{n}\left(X_{0}, \ldots, X_{n}\right), \quad n \geq 0,
\end{aligned}
$$

where $\left\{Y_{n}, n \geq 0\right\}$ is called as the generalized gambling system or the generalized random selection system. Let $\delta_{i}(j)$ be the Kronecker delta function on $S$, that is for $i, j \in S$

$$
\delta_{i}(j)= \begin{cases}0, & i \neq j \\ 1, & i=j\end{cases}
$$

We can obtain the following definition:

Definition 1. Let $\left\{Y_{n}, n \geq 0\right\}$ be a generalized random selection system defined as (8), $\left\{\sigma_{n}(\omega)\right.$, $n \geq 0\}$ be a nonnegative increasing stochastic sequence. We call

$$
S_{\left[\sigma_{n}(\omega)\right]}(\omega)=-\left(1 / \sum_{k=m}^{\left[\sigma_{n}(\omega)\right]} Y_{k}\right)\left[Y_{0} \log q\left(X_{0}, \ldots, X_{m-1}\right)+\sum_{k=m}^{\left[\sigma_{n}(\omega)\right]} Y_{k} \log p_{k}\left(X_{k} \mid X_{k-m}, \ldots, X_{k-1}\right)\right] .
$$

the relative entropy density of $m$ th-order nonhomogeneous Markov information source $\left\{X_{i}\right.$, $\left.0 \leq i \leq\left[\sigma_{n}(\omega)\right]\right\}$ on the generalized random selection system, where $\left[\sigma_{n}(\omega)\right]$ represents the integral part of $\sigma_{n}(\omega)$. Obviously, the generalized relative entropy density $S_{\left[\sigma_{n}(\omega)\right]}(\omega)$ is just the general relative entropy density $f_{n}(\omega)$ if $\sigma_{n}(\omega)=n, Y_{n} \equiv 1, n \geq 0$.

Definition 2. Let

$$
\begin{gathered}
h_{k}\left(x_{k-m}, \ldots, x_{k-1}\right)=-\sum_{x_{k} \in S} p_{k}\left(x_{k} \mid x_{k-m}, \ldots, x_{k-1}\right) \log p_{k}\left(x_{k} \mid x_{k-m}, \ldots, x_{k-1}\right), \\
H\left(p_{k}\left(s_{1} \mid X_{k-m}^{k-1}\right), \ldots, p_{k}\left(s_{M} \mid X_{k-m}^{k-1}\right)\right)=h_{k}\left(X_{k-m}, \ldots, X_{k-1}\right), \quad k \geq m .
\end{gathered}
$$

$H\left(p_{k}\left(s_{1} \mid X_{k-m}^{k-1}\right), \ldots, p_{k}\left(s_{M} \mid X_{k-m}^{k-1}\right)\right)$ is called the random conditional entropy of $X_{k}$ with respect to $X_{k-m}, \ldots, X_{k-1}$.

We denote $X^{n}=\left\{X_{0}, \ldots, X_{n}\right\}, X_{m}^{n}=\left\{X_{m}, \ldots, X_{n}\right\} . x^{n}, x_{m}^{n}$ the realization of $X^{n}, X_{m}^{n}$. 


\section{Main results and the proof}

Theorem 1. Let $\left\{X_{n}, n \geq 0\right\}$ be an mth-order nonhomogeneous Markov chain with the $m$ dimensional initial distribution (3) and the mth-order transition matrices (5). $S_{\left[\sigma_{n}(\omega)\right]}(\omega)$ and $H\left(p_{k}\left(s_{1} \mid X_{k-m}^{k-1}\right), \ldots, p_{k}\left(s_{M} \mid X_{k-m}^{k-1}\right)\right)$ are defined by (9) and (11), respectively. Denote

$$
D(\omega)=\left\{\omega: \lim _{n \rightarrow \infty} \sigma_{n}(\omega)=\infty, \quad 0<\limsup _{n \rightarrow \infty}\left(\sigma_{n}(\omega) / \sum_{k=m}^{\left[\sigma_{n}(\omega)\right]} Y_{k}\right) \leq M_{o}\right\},
$$

then

$$
\begin{array}{r}
\lim _{n \rightarrow \infty}\left[S_{\left[\sigma_{n}(\omega)\right]}(\omega)-\frac{1}{\sum_{k=m}^{\left[\sigma_{n}(\omega)\right]} Y_{k}} \sum_{k=m}^{\left[\sigma_{n}(\omega)\right]} Y_{k} H\left(p_{k}\left(s_{1} \mid X_{k-m}^{k-1}\right), \ldots, p_{k}\left(s_{M} \mid X_{k-m}^{k-1}\right)\right)\right]=0 . \\
P-\text { a.s. } \omega \in D(\omega) .
\end{array}
$$

Proof. On the probability space $(\Omega, \mathscr{F}, \mathscr{P})$, let $\lambda$ be a constant, $\delta_{i}(j)$ be Kronecker function. Denote $g_{k}(j)=-\log p_{k}\left(j \mid X_{k-m}^{k-1}\right)$, we construct the following product distribution:

$\mu\left(x_{0}, \ldots x_{n} ; \lambda\right)=q\left(x_{0}, \ldots, x_{m-1}\right) \prod_{k=m}^{n} \exp \left\{\lambda y_{k} g_{k}(j) \delta_{j}\left(x_{k}\right)\right\}\left[\frac{p_{k}\left(x_{k} \mid x_{k-m}^{k-1}\right)}{1+\left(e^{\lambda y_{k} g_{k}(j)}-1\right) p_{k}\left(j \mid x_{k-m}^{k-1}\right)}\right], \quad n \geq m$.

Where

$$
y_{k}=f_{k-1}\left(x_{0}, \ldots x_{k-1}\right), \quad k \geq 1 \text {. }
$$

By (14), we have

$$
\begin{aligned}
& \sum_{x_{n} \in S} \mu\left(x_{0}, \ldots x_{n}, \lambda\right)=\sum_{x_{n} \in S} q\left(x_{0}, \ldots, x_{m-1}\right) \prod_{k=m}^{n} \exp \left\{\lambda g_{k}(j) y_{k} \delta_{j}\left(x_{k}\right)\right\}\left[\frac{p_{k}\left(x_{k} \mid x_{k-m}^{k-1}\right)}{1+\left(e^{\lambda g_{k}(j) y_{k}}-1\right) p_{k}\left(j \mid x_{k-m}^{k-1}\right)}\right] \\
& =\mu\left(x_{0}, \ldots x_{n-1} ; \lambda\right) \sum_{x_{n} \in S} \exp \left\{\lambda y_{n} g_{n}(j) \delta_{j}\left(x_{n}\right)\right\}\left[\frac{p_{n}\left(x_{n} \mid x_{n-m}^{n-1}\right)}{1+\left(e^{\lambda g_{n}(j) y_{n}}-1\right) p_{n}\left(j \mid x_{n-m}^{n-1}\right)}\right] \\
& =\mu\left(x_{0}, \ldots x_{n-1} ; \lambda\right) \frac{1}{1+\left(e^{\lambda g_{n}(j) y_{n}}-1\right) p_{n}\left(j \mid x_{n-m}^{n-1}\right)}\left[\sum_{x_{n}=j}+\sum_{x_{n} \neq j}\right] \\
& =\mu\left(x_{0}, \ldots x_{n-1} ; \lambda\right) \frac{e^{\lambda g_{n}(j) y_{n}} p_{n}\left(j \mid x_{n-m}^{n-1}\right)+1-p_{n}\left(j \mid x_{n-m}^{n-1}\right)}{1+\left(e^{\lambda g_{n}(j) y_{n}}-1\right) p_{n}\left(j \mid x_{n-m}^{n-1}\right)} \\
& =\mu\left(x_{0}, \ldots x_{n-1} ; \lambda\right) \text {. }
\end{aligned}
$$

Therefore $\mu\left(x_{0}, \ldots x_{n} ; \lambda\right), n=1,2, \ldots$ are a family of consistent distribution functions on $S^{n+1}$. Let

$$
U_{n}(\lambda, \omega)=\frac{\mu\left(X_{0}, \ldots X_{n} ; \lambda\right)}{p\left(X_{0}, \ldots, X_{n}\right)}
$$

By (6), (14) and (16), we have

$$
U_{n}(\lambda, \omega)=\exp \left\{\sum_{k=m}^{n} \lambda Y_{k} g_{k}(j) \delta_{j}\left(X_{k}\right)\right\} \prod_{k=m}^{n}\left[\frac{1}{1+\left(e^{\lambda Y_{k} g_{k}(j)}-1\right) p_{k}\left(j \mid X_{k-m}^{k-1}\right)}\right], \quad n \geq m .
$$


It is easy to see that $U_{n}(\lambda, \omega)$ is a nonnegative sup-martingale from Doob's martingale convergence theorem (see [14]). Therefore,

$$
\lim _{n \rightarrow \infty} U_{n}(\lambda, \omega)=U_{\infty}(\lambda, \omega)<\infty . \quad P-\text { a.s. }
$$

By (12), (18) we have

$$
\limsup _{n \rightarrow \infty} \frac{1}{\sum_{k=m}^{\left[\sigma_{n}(\omega)\right]} Y_{k}} \log U_{\left[\sigma_{n}(\omega)\right]}(\lambda, \omega) \leq 0 . \quad P-\text { a.s. } \quad \omega \in D(\omega)
$$

By (17) and (19) we have

$$
\begin{gathered}
\limsup _{n \rightarrow \infty}\left\{\frac{1}{\sum_{k=m}^{\left[\sigma_{n}(\omega)\right]} Y_{k}} \sum_{k=m}^{\left[\sigma_{n}(\omega)\right]} \lambda Y_{k} g_{k}(j) \delta_{j}\left(X_{k}\right)\right. \\
\left.-\frac{1}{\sum_{k=m}^{\left[\sigma_{n}(\omega)\right]} Y_{k}} \sum_{k=m}^{\left[\sigma_{n}(\omega)\right]} \log \left[1+\left(e^{\lambda Y_{k} g_{k}(j)}-1\right) p_{k}\left(j \mid X_{k-m}^{k-1}\right)\right]\right\} \leq 0 . \\
P-\text { a.s. } \omega \in D(\omega)
\end{gathered}
$$

By (20), the inequalities $1-1 / x \leq \ln x \leq x-1,(x>0), e^{x}-1-x \leq(1 / 2) x^{2} e^{|x|}$ and the properties of superior limit

$$
\limsup _{n \rightarrow \infty}\left(a_{n}-b_{n}\right) \leq 0 \Rightarrow \limsup _{n \rightarrow \infty}\left(a_{n}-c_{n}\right) \leq \limsup _{n \rightarrow \infty}\left(b_{n}-c_{n}\right)
$$

we have

$$
\begin{aligned}
& \limsup _{n \rightarrow \infty} \frac{1}{\sum_{k=m}^{\left[\sigma_{n}\right]} Y_{k}} \sum_{k=m}^{\left[\sigma_{n}\right]} \lambda Y_{k}\left\{g_{k}(j) \delta_{j}\left(X_{k}\right)-g_{k}(j) p_{k}\left(j \mid X_{k-m}^{k-1}\right)\right\} \\
& \quad \leq \limsup _{n \rightarrow \infty} \frac{1}{\sum_{k=m}^{\left[\sigma_{n}\right]} Y_{k}} \sum_{k=m}^{\left[\sigma_{n}\right]}\left\{\log \left[1+\left(e^{\lambda Y_{k} g_{k}(j)}-1\right) p_{k}\left(j \mid X_{k-m}^{k-1}\right)\right]-\lambda Y_{k} g_{k}(j) p_{k}\left(j \mid X_{k-m}^{k-1}\right)\right\} \\
& \quad \leq \limsup _{n \rightarrow \infty} \frac{1}{\sum_{k=m}^{\left[\sigma_{n}\right]} Y_{k}} \sum_{k=m}^{\left[\sigma_{n}\right]} p_{k}\left(j \mid X_{k-m}^{k-1}\right)\left[e^{\lambda Y_{k} g_{k}(j)}-1-\lambda Y_{k} g_{k}(j)\right] \\
& \quad \leq\left(\lambda^{2} / 2\right) \limsup _{n \rightarrow \infty} \frac{1}{\sum_{k=m}^{\left[\sigma_{n}\right]} Y_{k}} \sum_{k=m}^{\left[\sigma_{n}\right]} p_{k}\left(j \mid X_{k-m}^{k-1}\right) g_{k}^{2}(j) Y_{k}^{2} e^{\left|\lambda Y_{k} g_{k}(j)\right|} \\
& \quad=\left(\lambda^{2} / 2\right) \limsup _{n \rightarrow \infty} \frac{1}{\sum_{k=m}^{\left[\sigma_{n}\right]} Y_{k}} \sum_{k=m}^{\left[\sigma_{n}\right]} Y_{k}^{2} \log ^{2} p_{k}\left(j \mid X_{k-m}^{k-1}\right) \cdot p_{k}\left(j \mid X_{k-m}^{k-1}\right)^{1-\left|\lambda Y_{k}\right|} .
\end{aligned}
$$

Noticing that $\alpha=\max \{|a|,|b|\}$ exists and $\left|Y_{k}\right| \leq \alpha$, taking $0<\lambda<1 / \alpha$, dividing both sides of (21) by $\lambda$, we have

$$
\limsup _{n \rightarrow \infty} \frac{1}{\sum_{k=m}^{\left[\sigma_{n}\right]} Y_{k}} \sum_{k=m}^{\left[\sigma_{n}\right]} Y_{k}\left\{g_{k}(j) \delta_{j}\left(X_{k}\right)-g_{k}(j) p_{k}\left(j \mid X_{k-m}^{k-1}\right)\right\}
$$




$$
\begin{gathered}
\leq \frac{\lambda}{2} \limsup _{n \rightarrow \infty} \frac{1}{\sum_{k=m}^{\left[\sigma_{n}\right]} Y_{k}} \sum_{k=m}^{\left[\sigma_{n}\right]} Y_{k}^{2} \log ^{2} p_{k}\left(j \mid X_{k-m}^{k-1}\right) \cdot p_{k}\left(j \mid X_{k-m}^{k-1}\right)^{1-\lambda\left|Y_{k}\right|} \\
\leq \frac{\lambda \alpha}{2} \limsup _{n \rightarrow \infty} \frac{1}{\sum_{k=m}^{\left[\sigma_{n}\right]} Y_{k}} \sum_{k=m}^{\left[\sigma_{n}\right]}\left|Y_{k}\right| \log ^{2} p_{k}\left(j \mid X_{k-m}^{k-1}\right) \cdot p_{k}\left(j \mid X_{k-m}^{k-1}\right)^{1-\lambda \alpha} . \\
P-\text { a.s. } \omega \in D(\omega) .
\end{gathered}
$$

Consider the function

$$
\phi(x)=(\log x)^{2} x^{1-\lambda}, \quad 0<x \leq 1,0<\lambda<1 .(\operatorname{set} \phi(0)=0)
$$

Letting

$$
\phi^{\prime}(x)=x^{-\lambda}\left[2(\log x)+(1-\lambda)(\log x)^{2}\right]=0,
$$

it can be concluded that on the internal $[0,1]$,

$$
\max \{\phi(x), 0 \leq x \leq 1\}=\phi\left(e^{2 /(\lambda-1)}\right)=\left(\frac{2}{\lambda-1}\right)^{2} e^{-2} .
$$

By (22), (23) and (24), in the case $0<\lambda<1 / \alpha$, we have

$$
\begin{aligned}
& \limsup _{n \rightarrow \infty} \frac{1}{\sum_{k=m}^{\left[\sigma_{n}\right]} Y_{k}} \sum_{k=m}^{\left[\sigma_{n}(\omega)\right]} Y_{k}\left\{g_{k}(j) \delta_{j}\left(X_{k}\right)-g_{k}(j) p_{k}\left(j \mid X_{k-m}^{k-1}\right)\right\} \\
& \quad \leq \frac{\lambda \alpha}{2} \limsup _{n \rightarrow \infty} \frac{1}{\sum_{k=m}^{\left[\sigma_{n}\right]} Y_{k}} \sum_{k=m}^{\left[\sigma_{n}(\omega)\right]}\left|Y_{k}\right|\left(\frac{2}{\lambda \alpha-1}\right)^{2} e^{-2} \\
& \quad \leq \frac{2 \lambda \alpha e^{-2}}{(1-\lambda \alpha)^{2}} \limsup _{n \rightarrow \infty}\left(\sum_{k=m}^{\left[\sigma_{n}(\omega)\right]}\left|Y_{k}\right| / \sum_{k=m}^{\left[\sigma_{n}(\omega)\right]} Y_{k}\right) . \quad P-\text { a.s. } \quad \omega \in D(\omega)
\end{aligned}
$$

By (12) we have

$$
\begin{aligned}
& \limsup _{n \rightarrow \infty}\left(\sum_{k=m}^{\left[\sigma_{n}(\omega)\right]}\left|Y_{k}\right| / \sum_{k=m}^{\left[\sigma_{n}(\omega)\right]} Y_{k}\right) \leq \limsup _{n \rightarrow \infty}\left(\sum_{k=m}^{\left[\sigma_{n}(\omega)\right]} \alpha / \sum_{k=m}^{\left[\sigma_{n}(\omega)\right]} Y_{k}\right) \\
& \leq \limsup _{n \rightarrow \infty}\left(\alpha\left(\sigma_{n}(\omega)-m+1\right) / \sum_{k=m}^{\left[\sigma_{n}(\omega)\right]} Y_{k}\right) \leq \limsup _{n \rightarrow \infty}\left(\alpha \cdot \sigma_{n}(\omega) / \sum_{k=m}^{\left[\sigma_{n}(\omega)\right]} Y_{k}\right) \leq \alpha M_{o} . \\
& P-\text { a.s. } \quad \omega \in D(\omega)
\end{aligned}
$$

It follows from (25) and (26) that

$$
\begin{aligned}
& \limsup _{n \rightarrow \infty} \frac{1}{\sum_{k=m}^{\left[\sigma_{n}\right]} Y_{k}} \sum_{k=m}^{\left[\sigma_{n}(\omega)\right]} Y_{k}\left\{g_{k}(j) \delta_{j}\left(X_{k}\right)-g_{k}(j) p_{k}\left(j \mid X_{k-m}^{k-1}\right)\right\} \leq \frac{2 \lambda \alpha^{2} e^{-2} M_{o}}{(1-\lambda \alpha)^{2}} . \\
& P-\text { a.s. } \quad \omega \in D(\omega)
\end{aligned}
$$


We choose $0<\lambda_{i}<1 / \alpha(i=1,2, \ldots)$ such that $\lambda_{i} \rightarrow 0+(i \rightarrow \infty)$, it follows from (27) that $\limsup _{n \rightarrow \infty} \frac{1}{\sum_{k=m}^{\left[\sigma_{n}\right]} Y_{k}} \sum_{k=m}^{\left[\sigma_{n}\right]} Y_{k}\left\{g_{k}(j) \delta_{j}\left(X_{k}\right)-g_{k}(j) p_{k}\left(j \mid X_{k-m}^{k-1}\right)\right\} \leq 0 . \quad P-$ a.s. $\quad \omega \in D(\omega)$

It follows from (9), (10), (11), (28) and $g_{k}(j)=-\log p_{k}\left(j \mid X_{k-m}^{k-1}\right)$ that

$$
\begin{aligned}
& \limsup _{n \rightarrow \infty}\left[S_{\left[\sigma_{n}\right]}(\omega)-\frac{1}{\sum_{k=m}^{\left[\sigma_{n}\right]} Y_{k}} \sum_{k=m}^{\left[\sigma_{n}\right]} Y_{k} H\left(p_{k}\left(s_{1} \mid X_{k-m}^{k-1}\right), \ldots, p_{k}\left(s_{M} \mid X_{k-m}^{k-1}\right)\right)\right] \\
& \quad=\limsup _{n \rightarrow \infty} \frac{1}{\sum_{k=m}^{\left[\sigma_{n}\right]} Y_{k}} \sum_{k=m}^{\left[\sigma_{n}\right]} Y_{k}\left[-\log p_{k}\left(X_{k} \mid X_{k-m}^{k-1}\right)-E\left(-\log p_{k}\left(X_{k} \mid X_{k-m}^{k-1}\right) \mid X_{k-m}^{k-1}\right)\right] \\
& \quad=\limsup _{n \rightarrow \infty} \frac{1}{\sum_{k=m}^{\left[\sigma_{n}\right]} Y_{k}} \sum_{k=m}^{\left[\sigma_{n}\right]} \sum_{j=s_{1}}^{s_{M}} Y_{k}\left[g_{k}(j) \delta_{j}\left(X_{k}\right)-g_{k}(j) p_{k}\left(j \mid X_{k-m}^{k-1}\right)\right] \\
& \quad \leq \sum_{j=s_{1}}^{s_{M}} \limsup _{n \rightarrow \infty} \frac{1}{\sum_{k=m}^{\left[\sigma_{n}\right]} Y_{k}} \sum_{k=m}^{\left[\sigma_{n}\right]} Y_{k}\left[g_{k}(j) \delta_{j}\left(X_{k}\right)-g_{k}(j) p_{k}\left(j \mid X_{k-m}^{k-1}\right)\right] \\
& \quad \leq 0 .
\end{aligned}
$$

Take $-1 / \alpha<\lambda<0$, it follows from (21) that

$$
\begin{aligned}
\liminf _{n \rightarrow \infty} \frac{1}{\sum_{k=m}^{\left[\sigma_{n}\right]} Y_{k}} \sum_{k=m}^{\left[\sigma_{n}\right]} Y_{k}\left\{g_{k}(j) \delta_{j}\left(X_{k}\right)-g_{k}(j) p_{k}\left(j \mid X_{k-m}^{k-1}\right)\right\} \\
\geq \frac{\lambda}{2} \limsup _{n \rightarrow \infty} \frac{1}{\sum_{k=m}^{\left[\sigma_{n}\right]} Y_{k}} \sum_{k=m}^{\left[\sigma_{n}\right]} Y_{k}^{2} \log ^{2} p_{k}\left(j \mid X_{k-m}^{k-1}\right) \cdot p_{k}\left(j \mid X_{k-m}^{k-1}\right)^{1+\lambda\left|Y_{k}\right|} \\
\geq \frac{\lambda \alpha}{2} \limsup _{n \rightarrow \infty} \frac{1}{\sum_{k=m}^{\left[\sigma_{n}\right]} Y_{k}} \sum_{k=m}^{\left[\sigma_{n}\right]}\left|Y_{k}\right| \log ^{2} p_{k}\left(j \mid X_{k-m}^{k-1}\right) \cdot p_{k}\left(j \mid X_{k-m}^{k-1}\right)^{1+\lambda \alpha} . \\
P-\text { a.s. } \quad \omega \in D(\omega)
\end{aligned}
$$

We have by (23), (24), (26) and (30) that

$$
\begin{gathered}
\liminf _{n \rightarrow \infty} \frac{1}{\sum_{k=m}^{\left[\sigma_{n}\right]} Y_{k}} \sum_{k=m}^{\left[\sigma_{n}\right]} Y_{k}\left\{g_{k}(j) \delta_{j}\left(X_{k}\right)-g_{k}(j) p_{k}\left(j \mid X_{k-m}^{k-1}\right)\right\} \\
\geq \frac{\lambda \alpha}{2} \limsup _{n \rightarrow \infty} \frac{1}{\sum_{k=m}^{\left[\sigma_{n}\right]} Y_{k}} \sum_{k=m}^{\left[\sigma_{n}\right]}\left|Y_{k}\right| \cdot\left(\frac{2}{1+\lambda \alpha}\right)^{2} e^{-2} \\
\geq \frac{2 \lambda \alpha^{2} e^{-2} M_{o}}{(1+\lambda \alpha)^{2}} . \quad P-\text { a.s. } \quad \omega \in D(\omega)
\end{gathered}
$$

We choose $-1 / \alpha<\lambda_{i}<0(i=1,2, \ldots)$ such that $\lambda_{i} \rightarrow 0-(i \rightarrow \infty)$, it follows from (31) that $\liminf _{n \rightarrow \infty} \frac{1}{\sum_{k=m}^{\left[\sigma_{n}\right]} Y_{k}} \sum_{k=m}^{\left[\sigma_{n}\right]} Y_{k}\left\{g_{k}(j) \delta_{j}\left(X_{k}\right)-g_{k}(j) p_{k}\left(j \mid X_{k-m}^{k-1}\right)\right\} \geq 0 . \quad P-$ a.s. $\quad \omega \in D(\omega)$ 
In a similar way, it follows from (9), (10), (11), (32) and $g_{k}(j)=-\log p_{k}\left(j \mid X_{k-m}^{k-1}\right)$ that

$$
\begin{aligned}
& \liminf _{n \rightarrow \infty}\left[S_{\left[\sigma_{n}\right]}(\omega)-\frac{1}{\sum_{k=m}^{\left[\sigma_{n}\right]} Y_{k}} \sum_{k=m}^{\left[\sigma_{n}\right]} Y_{k} H\left(p_{k}\left(s_{1} \mid X_{k-m}^{k-1}\right), \ldots, p_{k}\left(s_{M} \mid X_{k-m}^{k-1}\right)\right)\right] \\
& \quad=\liminf _{n \rightarrow \infty} \frac{1}{\sum_{k=m}^{\left[\sigma_{n}\right]} Y_{k}} \sum_{k=m}^{\left[\sigma_{n}\right]} Y_{k}\left[-\log p_{k}\left(X_{k} \mid X_{k-m}^{k-1}\right)-E\left(-\log p_{k}\left(X_{k} \mid X_{k-m}^{k-1}\right) \mid X_{k-m}^{k-1}\right)\right] \\
& \quad=\liminf _{n \rightarrow \infty} \frac{1}{\sum_{k=m}^{\left[\sigma_{n}\right]} Y_{k}} \sum_{k=m}^{\left[\sigma_{n}\right]} \sum_{j=s_{1}}^{s_{M}} Y_{k}\left[g_{k}(j) \delta_{j}\left(X_{k}\right)-g_{k}(j) p_{k}\left(j \mid X_{k-m}^{k-1}\right)\right] \\
& \geq \sum_{j=s_{1}}^{\liminf _{n \rightarrow \infty}} \frac{1}{\sum_{k=m}^{\left[\sigma_{n}\right]} Y_{k}} \sum_{k=m}^{\left[\sigma_{n}\right]} Y_{k}\left[g_{k}(j) \delta_{j}\left(X_{k}\right)-g_{k}(j) p_{k}\left(j \mid X_{k-m}^{k-1}\right)\right] \\
& \geq 0 .
\end{aligned}
$$

By (29) and (33) we have

$$
\lim _{n \rightarrow \infty}\left[S_{\left[\sigma_{n}\right]}(\omega)-\frac{1}{\sum_{k=m}^{\left[\sigma_{n}\right]} Y_{k}} \sum_{k=m}^{\left[\sigma_{n}\right]} Y_{k} H\left(p_{k}\left(s_{1} \mid X_{k-m}^{k-1}\right), \ldots, p_{k}\left(s_{M} \mid X_{k-m}^{k-1}\right)\right)\right]=0 . \quad P-\text { a.s. } \omega \in D(\omega)
$$

The proof is accomplished.

\section{Some Corollaries for Shannon-McMillan theorems}

Corollary 1 ([13]). Let $\left\{X_{n}, n \geq 0\right\}$ be an mth-order nonhomogeneous Markov chain with the $m$ dimensional initial distribution (3) and the mth-order transition matrices (5), $f_{n}(\omega)$ and $H\left[p_{k}\left(s_{1} \mid X_{k-m}^{k-1}\right), \ldots, p_{k}\left(s_{M} \mid X_{k-m}^{k-1}\right)\right]$ be defined by (7) and (11), respectively. Then

$$
\lim _{n \rightarrow \infty}\left\{f_{n}(\omega)-\frac{1}{n} \sum_{k=m}^{n} H\left[p_{k}\left(s_{1} \mid X_{k-m}^{k-1}\right), \ldots, p_{k}\left(s_{M} \mid X_{k-m}^{k-1}\right)\right]\right\}=0 . \quad P-\text { a.s. }
$$

Proof. In Theorem 1 letting $\sigma_{n}(\omega)=n, Y_{n} \equiv 1, n \geq 0$, we obtain $S_{\left[\sigma_{n}\right]}(\omega)=f_{n}(\omega)$,

$$
\limsup _{n \rightarrow \infty}\left(\sigma_{n}(\omega) / \sum_{k=m}^{\left[\sigma_{n}(\omega)\right]} Y_{k}\right)=\limsup _{n \rightarrow \infty}\left(n / \sum_{k=m}^{n} Y_{k}\right)=\limsup _{n \rightarrow \infty} \frac{n}{n-m+1}=1 .
$$

Hence $D(\omega)=\Omega$. (35) follows from (13) immediately.

Corollary 2 ([7]). Let $\left\{X_{n}, n \geq 0\right\}$ be a nonhomogeneous Markov chain, denote

$$
\begin{gathered}
f_{n}(\omega)=-\frac{1}{n+1}\left[\log p\left(X_{0}\right)+\sum_{k=1}^{n} \log p_{k}\left(X_{k} \mid X_{k-1}\right)\right], \\
H\left(p_{k}\left(s_{1} \mid X_{k-1}\right), \ldots, p_{k}\left(s_{M} \mid X_{k-1}\right)\right)=-\sum_{x_{k} \in S} p_{k}\left(x_{k} \mid X_{k-1}\right) \log p_{k}\left(x_{k} \mid X_{k-1}\right) .
\end{gathered}
$$

Then

$$
\lim _{n \rightarrow \infty}\left\{f_{n}(\omega)-\frac{1}{n} \sum_{k=1}^{n} H\left[p_{k}\left(s_{1} \mid X_{k-1}\right), \ldots, p_{k}\left(s_{M} \mid X_{k-1}\right)\right]\right\}=0 . \quad P-\text { a.s. }
$$

Proof. Letting $m=1$ in Corollary 1, (37) follows from (35) directly. 


\section{References}

[1] C. Shannon, A mathematical theory of communication, Bell Syst. Tech. J., 27(1948), 379-423.

[2] B. Mcmillan, The Basic Theorem of information theory, Ann. Math. Statist., 24(1953), 196-216.

[3] L. Breiman, The individual ergodic theorem of information theory, Ann. Math. Statist., 28(1957), 809-811.

[4] K. L. Chung, The ergodic theorem of information theory, Ann. Math. Statist., 32(1961), 612-614.

[5] A. R. Barron, The strong ergodic theorem for densities:Generalized Shannon-McMillan-Breiman theorem, Ann. Probab., 13(1985), 1292-1303.

[6] P. H. Algoet, and T. M. Cover, A sandwich proof of Shannon-Mcmillan theorem, Ann. Probab., 16(1988), 899909.

[7] W. Liu and W. G. Yang, An extension of Shannon-Mcmillan theorem and some limit properties for nonhomogeneous Markov chains, Stochastic. Process. Appl., 61(1996), 129-145.

[8] P. Billingsley, Probability and Measure. Wiley, New York, 1986.

[9] R. V. Mises, Mathematical Theory of Probability and Statistics. Academic Press. New York, 1964.

[10] A. N. Kolmogorov, On the logical foundation of probability theory. Lecture Notes in Mathematics. SpringerVerlag. New York, 1982.1021:1-2.

[11] W. Liu, and Z. Wang, An extension of a theorem on gambling systems to arbitrary binary random variables, Statistics and Probability Letters, 28(1996), 51-58.

[12] Z. Wang, A strong limit theorem on random selection for the N-valued random variables, Pure and Applied Mathematics, 15(1999), 56-61.

[13] W. G. Yang and W. Liu, The asymptotic equipartition property for Mth-order nonhomogeneous Markov information source, IEEE Trans. Inform. Theory., 50(2004), 3326-3330.

[14] J. L. Doob, Stochastic Process. Wiley New York. 1953.

[15] Z. R. Xu and Y. J. Zhu, Flow controled quene with negative customers and preemptive priority, J. Jiangsu Univ. Sci-tech. Nat. Sci. 24(4)(2010), 400-404.

[16] K. K. Wang and D. C. Zong, A class of strong limit theorems for random sum of Cantor-like random sequence on gambling system, J. Jiangsu Univ. Sci-tech. Nat. Sci. 24(3) (2010), 305-308.

[17] Z. R. Xu and M. J. Li, Geo Geo 1 queue model with RCH strategy of negative customers and single vacation, J. Jiangsu Univ. Sci-tech. Nat. Sci., 25(2)(2011), 191-194, 2011.

[18] K. K. Wang and D. C. Zong, A class of strong deviation theorems on generalized gambling system for the sequence of arbitrary continuous random variables. J. Jiangsu Univ. Sci-tech. Nat. Sci., 25(2)(2011), 195-199.

[19] K. K. Wang, H. Ye and Y. Ma, A class of strong deviation theorems for multivariate function sequence of mthorder countable nonhomogeneous Markov chains, J. Jiangsu Univ. Sci-tech. Nat. Sci., 25(1)(2011), 93-96.

[20] K. K. Wang, A class of local strong limit theorems for Markov chains field on arbitrary Bethe tree, J. Jiangsu Univ. Sci-tech. Nat. Sci., 24 (2) (2010), 205-209.

[21] K. K. Wang and D. C. Zong, Strong limit theorems of mth-order nonhomogeneous Markov chains on fair gambling system. J. Jiangsu Univ. Sci-tech. Nat. Sci., 24(4)(201), 410-413.

[22] K. K. Wang and F. Li, Strong deviation theorems for the sequence of arbitrary random variables with respect to product distribution in random selection system, J. Jiangsu Univ. Sci-tech. Nat. Sci., 24(1)(2010), 91-94.

[23] K. K. Wang and F. Li, A class of Shannon-McMillan theorems for mth-order nonhomogeneous Markov information source on generalized gambling system, J. Jiangsu Univ. Sci-tech. Nat. Sci., 25 (4)(2011), 396-400.

[24] K. K. Wang, F. Li and Y. Ma, A class of Shannon-McMillan theorems for nonhomogeneous Markov information source on random selection system, J. Jiangsu Univ. Sci-tech. Nat. Sci., 25(3)(2011), 299-302.

[25] S. H. Li and Z. G. Zhou, Shrunken estimators of seemingly unrelated regression model, J. Jiangsu Univ. Sci-tech. Nat. Sci., 24(2)(2010), 197-200.

[26] Y. X. Yuan and J. S. Jiang, A direct updating method for the stiffness matrix, J. Jiangsu Univ. Sci-tech. Nat. Sci., 24 (2)(2010), 193-196.

[27] M. Hu, Feasibility of classification on two kinds of 2-person noncooperative finite games, J. Jiangsu Univ. Scitech. Nat. Sci., 24 (4) (2010), 405-409. 
[28] X. Q. Hua and D. P. Xu, Two groups of three-level high-precision explicit schemes for dispersive equation, J. Jiangsu Univ. Sci-tech. Nat. Sci., 24(4)(2010), 395-399.

[29] Y. X. Yuan and J. S. Jiang, Generalized inverse eigenvalue problems for tridiagonal symmetric matrices, J. Jiangsu Univ. Sci-tech. Nat. Sci., 24 (1) (2010), 88-90.

[30] J. E. Wu and T. C. Wu, Regularized Symmlq for solving Symm integral equation, J. Jiangsu Univ. Sci-tech. Nat. Sci., 25(3) (2011), 294-298. 2011

[31] S. H. Li and Z. G. Zhou, Linear minimax estimators of estimable function in multiple Gauss-Markov model with matrix loss function, J. Jiangsu Univ. Sci-tech. Nat. Sci., 24(1)(2010), 95-98.

[32] C. J. Fang, D. C. Zong, Strong deviation theorems for the stochastic sequence on the Poisson distribution in generalized gambling systems, J. Jiangsu Univ. Sci-tech. Nat. Sci., 26(1)(2012), 103-106.

[33] Z. S. Zang. The symmetric positive semidefinite solution of a class of matrices equation with a submatrix constraint, J. Jiangsu Univ. Sci-tech. Nat. Sci., 26(1)(2012), 100-102.

School of Mathematics and Physics, Jiangsu University of Science and Technology, Zhenjiang 212003, China.

E-mail: wkk.cn@126.com

Department of Computer Science, Changshu Institute of Technology, Changshu 215500, China.

E-mail: zongdecai@126.com 